\title{
The 12-month period prevalence and cardiac manifestations of HIV in patients with acute coronary syndrome at a tertiary hospital in Cape Town, South Africa: a retrospective cross-sectional study
}

\author{
Camilla Pennefather ${ }^{1}$, Tonya Esterhuizen ${ }^{2}$, Anton Doubell ${ }^{3}$ and Eric H. Decloedt ${ }^{1 *}$ (D)
}

\begin{abstract}
Background: HIV-positive patients are increasingly being affected by non-communicable diseases such as coronary artery disease (CAD). Data from high-income countries (HICS) indicate that HIV-positive patients have different riskfactor profiles for acute coronary syndrome (ACS) as well as different cardiac manifestations of this syndrome compared to HIV-negative patients. There is limited data from Sub-Saharan Africa (SSA), and particularly from South Africa with the biggest HIV epidemic in the world. The objective of this study was to determine the 12-month period prevalence of HIV in patients with ACS and to compare the risk-factor profile, ACS presentation and management between HIV-positive and HIV-negative adults.

Methods: We included all patients hospitalised with ACS from 01 January to 31 December 2018 in a tertiary hospital, Tygerberg Hospital, in Cape Town, South Africa. The HIV-status of all patients was determined using routine clinical records. We performed multiple conditional logistic regression on HIV-positive and HIV-negative patients (1:3 ratio) to compare the risk factor profile, ACS presentation and management between the groups.

Results: Among 889 patients, 30 (3.4\%) were HIV-positive (95\% confidence interval (CI): 2.3-4.8). HIV-positive patients were younger, more frequently men, and had a lower prevalence of medical comorbidities and a family history of CAD. They were more likely to present with ST-elevation myocardial infarction (STEMI) [odd's ratio (OR) (95\% Cl): 3.12 (1.2-8.4)], and have single-vessel disease [OR (95\% Cl): 3.03 (1.2-8.0)]. Angiographic and echocardiographic data, as well as management, did not differ between the groups. Among HIV-positive patients, 17 (65\%) were virally suppressed (HIV viral load $<200$ copies $/ \mathrm{mL}$ ) with a median CD4 ${ }^{+}$count of $271 \mathrm{cells} / \mathrm{mm}^{3}$. The majority $(20,67 \%)$ of HIV-positive patients were receiving antiretroviral therapy at the time of the ACS.
\end{abstract}

\footnotetext{
* Correspondence: ericdecloedt@sun.ac.za

'Division of Clinical Pharmacology, Department of Medicine, Faculty of Medicine and Health Sciences, Stellenbosch University and Tygerberg Hospital, PO Box 241, Cape Town 8000, Republic of South Africa

Full list of author information is available at the end of the article
}

(c) The Author(s). 2021 Open Access This article is licensed under a Creative Commons Attribution 4.0 International License, which permits use, sharing, adaptation, distribution and reproduction in any medium or format, as long as you give appropriate credit to the original author(s) and the source, provide a link to the Creative Commons licence, and indicate if changes were made. The images or other third party material in this article are included in the article's Creative Commons licence, unless indicated otherwise in a credit line to the material. If material is not included in the article's Creative Commons licence and your intended use is not permitted by statutory regulation or exceeds the permitted use, you will need to obtain permission directly from the copyright holder. To view a copy of this licence, visit http://creativecommons.org/licenses/by/4.0/ The Creative Commons Public Domain Dedication waiver (http://creativecommons.org/publicdomain/zero/1.0/) applies to the data made available in this article, unless otherwise stated in a credit line to the data. 
Conclusions: We found an HIV-prevalence of 3.4\% (95\% CI 2.3-4.8) in adults with ACS in a high endemic HIV region. HIV-positive patients were younger and more likely to present with STEMIs and single-vessel disease, but had fewer CAD risk factors, suggesting additional mechanisms for the development of ACS.

Keywords: Acute coronary syndrome, HIV infection, Risk factors, Cardiac manifestations, Prevalence

\section{Background}

The incidence of acute coronary syndrome (ACS) in HIV-positive individuals is increasing [1]. Antiretroviral therapy (ART) treated HIV-positive patients are experiencing fewer HIV-related opportunistic infections and are increasingly being affected by the same ageassociated spectrum of atherosclerotic disease, including coronary artery disease (CAD), as the general population [2]. Atherosclerotic disease, however, is emerging about 10 years earlier than in their uninfected counterparts, suggesting an alternative pathogenesis in HIV-positive patients [3]. CAD accounts for $8-22 \%$ of deaths among the HIV-positive population, making it an important cause of morbidity and mortality [4]. This is in contrast to the pre-ART era, when cardiomyopathies, pancarditis, conduction system abnormalities, pulmonary hypertension leading to heart failure, and neoplastic infiltration were more important cardiac manifestations of HIV infection [5].

Despite much data from Europe and North America, there are limited studies from South Africa, a low and middle-income country (LMIC), reporting on the prevalence of HIV in ACS, as well as the CAD risk factors, cardiac manifestations and management of ACS in these patients [6]. Most studies demonstrate HIV-positive patients with ACS to be younger, predominantly male, likely to smoke but with a lower prevalence of traditional CAD risk factors (diabetes mellitus, hypertension, dyslipidaemia) compared to their HIV-negative counterparts [1]. The increased incidence of ACS in the HIV-positive population suggests additional pathogenic mechanisms including increased survival time, ongoing HIV inflammation, immune-activation and ART-induced metabolic derangements $[4,7]$. Chronic HIV-associated inflammation is associated with abnormal lipid profiles independent of ART, unstable plaque morphology, and more severe coronary artery stenosis [8]. Furthermore, it has been shown that HIV-positive patients have increased C-reactive protein, interleukin-6 and D-dimer levels, contributing to a pro-inflammatory and pro-thrombotic environment [5]. HIV-replication and immuneactivation causes up-regulation of tissue factor pathways and chronic platelet activation, which may promote atherogenesis and put these patients at an increased risk of thrombotic events [7]. The clinical presentation of HIV-positive patients with CAD seems to be similar to that of the general population, and includes silent ischaemia, stable angina and ACS [7]. ACS is the main clinical presentation, of which ST-segment elevation myocardial infarction (STEMI) is the main sub-type. Non-ST-elevated myocardial infarction (NSTEMI) and unstable angina (UA) are the most common presentations of ACS in HIV-uninfected persons [1].

$\mathrm{CAD}$ is no longer a disease affecting just the highincome countries (HICs); LMICs today are experiencing a greater morbidity and mortality from ACS, with the death toll affecting the younger, productive ages more heavily [9]. This is evidenced by the shift from communicable diseases $(48.1 \%$ prevalence in $2005 ; 31.3 \%$ prevalence in $2016)$ to non-communicable diseases $(42.9 \%$ prevalence in 2005; $57.4 \%$ prevalence in 2016) as the primary cause of death in South Africans in the last decade [10]. Despite this, HIV still affects $13.1 \%$ of the South African population [11]. HIV-positive patients are hypothesised to be at an increased risk for CAD and the combined effect of these two distinct epidemics may have a marked impact on morbidity and life expectancy $[7,12]$.

We conducted a retrospective cross-sectional study to estimate the 12-month period prevalence of HIV in a South African population from Cape Town presenting with ACS. The second objective of the study aims to describe the CAD risk factors, cardiac manifestations, and management of HIV-positive patients in comparison to HIV-negative patients, using a case-control study design.

\section{Methods}

We conducted a retrospective review of all adult patients presenting to Tygerberg Hospital (TBH), Cape Town, from 01 January to 31 December 2018 with ACS. TBH is the largest public-sector tertiary referral hospital in the Cape Town Metropole and serves over 3.4 million people; mostly vulnerable populations from densely populated low-income communities and rural areas.

Patients were identified using clopidogrel prescription data from the JAC electronic dispensing system [13]. We used the Electronic Content Management System (ECM) to access stored medical records to identify patients with the diagnosis of ACS documented in their medical record as the indication for clopidogrel treatment. The diagnosis of myocardial infarction (MI) at Tygerberg Hospital is made according to the Fourth 
Universal Definition: acute myocardial injury with clinical evidence of acute myocardial ischaemia and a rise of cardiac troponin levels with at least one value $>99$ th percentile of the upper reference limit and at least one of the following: symptoms of myocardial ischaemia, new ischaemic changes on the ECG, pathological Q-waves on the ECG, imaging evidence of new loss of myocardium or new localised wall motion abnormality in a pattern indicative of ischaemic causes, presence of a coronary thrombus by angiography [14]. UA is diagnosed by the presence of ischaemic symptoms suggestive of ACS, without elevated biomarkers or ECG changes suggestive of infarction [14]. The diagnosis of ACS was confirmed by reviewing attending physician discharge summaries, and cross-checking the admission records, electrocardiograms (ECGs), troponin levels, echocardiograms and angiogram reports of each patient. Patients with repeated ACS-events within the year were only counted once, and their first ACS-event within the year was described.

We extracted the HIV status of all patients with a clinical diagnosis of ACS to determine the period prevalence. HIV data was obtained from the South African National Health Laboratory Service (NHLS) database. The NHLS is the only service provider of HIV testing in the public sector and an absent result implies that HIV testing was not done in the public sector. HIV-positive patients were classified as virally supressed $(\mathrm{VL}<200$ copies $/ \mathrm{mL}$ ) or virally unsuppressed (VL $>200$ copies/ $\mathrm{mL}$ ), and the number of immune-suppressed patients $\left(\mathrm{CD} 4^{+}\right.$count $<500$ cells $\left./ \mathrm{mm}^{3}\right)$ was described.
In the case-control analysis of our study we compared the CAD risk factors, ACS-type and management of HIV-positive compared with HIV-negative patients with ACS. In order to do this, we selected three HIV-negative patients (confirmed HIV-negative test result on the NHLS) for every HIV-positive patient (1:3 ratio) (Fig. 1). The HIV-negative patients were chosen by sequentially selecting the next three patients with a negative HIVtest following the HIV-positive patient, by date order of clopidogrel dispensing. Our method did not allow for matching according to baseline characteristics. We extracted the following CAD variables from their medical records on the ECM system: comorbidities (hypertension, dyslipidaemia, diabetes mellitus), family history of CAD, social risk factors (tobacco-smoking, body mass index $>25 \mathrm{~kg} / \mathrm{m}^{2}$, ethanol-misuse), the type of ACS (STEMI, NSTEMI and UA), the culprit coronary artery, the region of myocardium involved, single- or multivessel coronary artery disease, echocardiographic findings, pharmacological and interventional management of the patients, as well as their serum creatinine concentration on presentation. We identified medical comorbidities using discharge summary diagnoses, prescription data and medical records on the ECM. In the public sector, hypertension, diabetes mellitus and dyslipidaemia are diagnosed and managed according to the Adult Hospital Level Standard Treatment Guidelines and Essential Medicines List [15]. Diagnosis of hypertension requires at least 3 blood pressure readings $\geq 140 / 90$ at least 2 days apart. Diagnosis of diabetes mellitus is made based on the following: symptoms of hyperglycaemia or metabolic

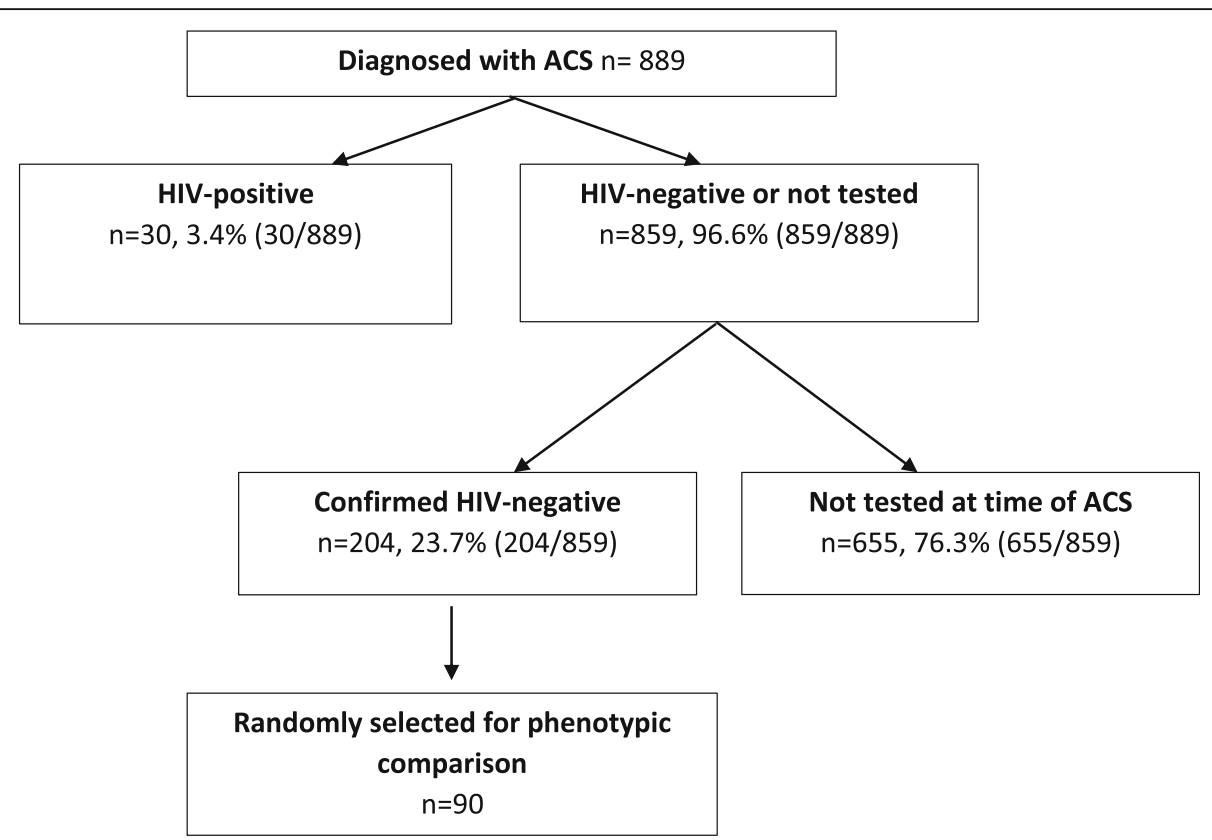

Fig. 1 Diagram of the selection of patients for study inclusion 
decompensation with any one single test that confirms a random plasma glucose $\geq 11.1 \mathrm{mmol} / \mathrm{L}$, fasting plasma glucose $\geq 7.0 \mathrm{mmol} / \mathrm{L}, \mathrm{HbA} 1 \mathrm{c} \geq 6.5 \%$ or 2 -h post-load glucose $\geq 11.1 \mathrm{mmol} / \mathrm{L}$. Dyslipidaemia is diagnosed based on the finding of abnormal serum lipid levels, and treatment thereof is determined by a cardiovascular risk score (taking into account total cholesterol, high-density lipoprotein (HDL), gender, age and smoking status) [15].

The calculation of the 12-month period prevalence of HIV-positive patients presenting to TBH with ACS in 2018 was determined from the entire 2018 sample using a simple proportion calculation. From this sample, a sub-sample consisting of just those infected with HIV (exposed) and three times the number of exposed with a confirmed negative HIV test (unexposed) were selected for further statistical analysis. Data were analysed using Stata version 11.0 (StatCorp, College Station, TX, USA). Baseline characteristics of exposed and unexposed patients were compared by chi-square tests or 2-sided Fisher's exact tests for categorical variables and T-tests or Wilcoxon rank-sum tests for continuous variables. Normally distributed data were described using means, medians and standard deviations (SD). 95\% confidence intervals (CIs), odds ratios (ORs), and $p$-values were derived for all appropriate data. Logistic regression was performed, controlling for age and sex, in order to determine associations between risk factor profile/cardiac manifestations in those presenting with ACS and HIV- status. Adjusted 95\% CIs, ORs and $p$-values were derived after such adjustment. Statistical significance was set at a $p$-value of less than 0.05 .

Ethical approval for the study as well as a waiver of consent was obtained from the Human Research Ethics Committee of the Faculty of Medicine and Health Sciences of Stellenbosch University (Reference number: U19/05/023).

\section{Results}

\section{Prevalence of HIV in ACS}

We identified 889 patients who presented to TBH in 2018 with the diagnosis of ACS of which 30 patients were HIV-positive to give a 12 month period prevalence of 3.4\% (95\% CI 2.3-4.8) (Fig. 1).

\section{Baseline characteristics}

Thirty HIV-positive ACS patients were compared with 90 randomly selected HIV-negative ACS patients, with the following findings (Table 1): The HIV-positive patients were on average 7 years younger (50 years compared with 57 years), more frequently men $(n=20 / 30$; $67 \%$ compared with $n=50 / 90 ; 56 \%$ ), had a lower prevalence of medical comorbidities (hypertension, dyslipidaemia and diabetes mellitus) ( $n=20 / 30 ; 67 \%$ compared with $n=83 / 90$; 92\%), and a lower prevalence of a family history of CAD ( $n=2 / 30 ; 7 \%$ compared with $n=18 / 90$; $20 \%)$, even after adjusting for age and sex. There was no

Table 1 Characteristics of HIV-positive compared to HIV-negative patients (crude and adjusted)

\begin{tabular}{|c|c|c|c|c|c|}
\hline Characteristic, n (\%) except where specified & $\mathrm{HIV}+(n=30)$ & HIV- $(n=90)$ & $\begin{array}{l}\text { Crude OR } \\
(95 \% \mathrm{Cl})\end{array}$ & $\begin{array}{l}{ }^{a} \mathrm{aOR} \\
(95 \% \mathrm{Cl})\end{array}$ & adjusted $p$-value \\
\hline \multicolumn{6}{|l|}{ Demographics } \\
\hline Age (years), mean (SD) & $50(11.90)$ & $57.32(11.63)$ & $0.95(0.91-0.98)$ & & $0.008^{\#}$ \\
\hline Male & $20(66.7 \%)$ & $50(55.6 \%)$ & $1.60(0.67-3.80)$ & & $0.586^{\#}$ \\
\hline \multicolumn{6}{|l|}{ Cardiovascular risk factors } \\
\hline Medical comorbidities & $20(66.7 \%)$ & $83(92.2 \%)$ & $0.17(0.06-0.50)$ & $0.21(0.07-0.65)$ & 0.007 \\
\hline Hypertension & $13(43.3 \%)$ & $64(71.1 \%)$ & $0.31(0.13-0.73)$ & $0.39(0.16-0.97)$ & 0.044 \\
\hline Dyslipidaemia & $7(23.3 \%)$ & $45(50.0 \%)$ & $0.30(0.12-0.78)$ & $0.28(0.11-0.76)$ & 0.012 \\
\hline Diabetes mellitus & $4(13.3 \%)$ & $34(37.8 \%)$ & $0.25(0.08-0.79)$ & $0.31(0.10-1.00)$ & 0.051 \\
\hline Personal history CAD & $4(13.3 \%)$ & 27 (30.0\%) & $0.36(0.11-1.13)$ & $0.48(0.15-1.60)$ & 0.234 \\
\hline Personal history CVA & $3(10.0 \%)$ & $10(11.1 \%)$ & $0.89(0.23-3.47)$ & $0.70(0.17-2.93)$ & 0.630 \\
\hline PVD & $3(10.0 \%)$ & $10(11.1 \%)$ & $0.89(0.23-3.47)$ & $1.08(0.26-4.51)$ & 0.913 \\
\hline Family history CAD & $2(6.7 \%)$ & $18(20.0 \%)$ & $0.29(0.06-1.31)$ & $0.20(0.04-0.99)$ & 0.048 \\
\hline Social risk factors & 25 (83.3\%) & 74 (82.2\%) & $1.08(0.36-3.25)$ & $0.42(0.11-1.55)$ & 0.192 \\
\hline Smoking & $23(76.7 \%)$ & $67(74.4 \%)$ & $1.13(0.43-2.97)$ & $0.57(0.18-1.76)$ & 0.329 \\
\hline $\mathrm{BMI}>25 \mathrm{~kg} / \mathrm{m}^{2}$ & 7 (23.3\%) & 29 (32.2\%) & $0.64(0.25-1.66)$ & $0.56(0.21-1.50)$ & 0.249 \\
\hline Ethanol-misuse & $3(10.0 \%)$ & $10(11.1 \%)$ & $0.89(0.23-3.47)$ & $0.60(0.14-2.45)$ & 0.472 \\
\hline Serum creatinine $(\mu \mathrm{mol} / \mathrm{L})$, median $(\mathrm{IQR})$ & $71.5(59-98)$ & $82.5(66-103)$ & $0.99(0.97-1.00)$ & $0.99(0.98-1.01)$ & 0.361 \\
\hline
\end{tabular}

OR odds ratio, $C I$ confidence interval, $S D$ standard deviation, CAD coronary artery disease, CVA cerebrovascular accident, $P V D$ peripheral vascular disease, $B M I$ body mass index, IQR inter-quartile range

adjusted odds ratio conditioned on age and sex. ${ }^{*}$ crude $p$-value. Bolded text $=$ statistically significant 
difference in the social risk factor profile of the patients between the two groups. Although statistically nonsignificant, mention is made of the $11 \mu \mathrm{mol} / \mathrm{L}$ higher median serum creatinine in the HIV-negative sub-group.

\section{Acute coronary syndrome information}

Twenty-three $(n=23 / 30 ; 76.6 \%)$ HIV-positive patients presented with a STEMI compared to $48(n=48 / 90$; 53.3\%) controls, with HIV-positive patients having 3.12 greater odds (95\% CI 1.2-8.4) of presenting with a STEMI after adjusting for age and sex (Table 2). Furthermore, HIV-positive patients had 3.03 greater odds (95\% CI 1.2-8.0) of having single-vessel disease after adjusting as above $(n=16 / 30 ; 53 \%$ compared with $n=$ 19/90; 21\%). Although statistically nonsignificant, mention can be made of the increased prevalence of left anterior descending (LAD) artery involvement in the HIVpositive group $(n=16 / 30 ; 53.3 \%)$ compared to the HIVnegative group ( $n=38 / 90 ; 42.2 \%)$, translating into more LAD-territory infarcts in the former (Table 3).

\section{Echocardiographic information}

There was no difference in the echocardiographic data between the two groups, with the exception of the left ventricular internal dimension at end-systole (LVIDs), which was statistically significantly smaller in the HIVpositive compared to the HIV-negative sub-group (3.37 $\mathrm{mm}$ compared with $3.96 \mathrm{~mm}$ ) (Table 4). The systolic function was equally impaired in both groups.

\section{HIV-specific information}

The median $\mathrm{CD}_{4}^{+}$count in those with available $\mathrm{CD} 4^{+}$ results in the HIV-positive sub-group was 271 cells $/ \mathrm{mm}^{3}$ (Table 5). Twenty-two patients $(n=22 / 27 ; 81.5 \%)$ had a $\mathrm{CD} 4^{+}$count $<500$ cells $/ \mathrm{mm}^{3}$, with a nadir $\mathrm{CD} 4^{+}$count of 41 cells $/ \mathrm{mm}^{3}$. Seventeen $(n=17 / 26 ; 65.4 \%)$ patients were virally suppressed (HIV VL $<200$ copies/mL) and 13 $(n=13 / 26 ; 50 \%)$ had viral loads lower than detectable limits (HIV VL $<20$ copies $/ \mathrm{mL}$ ). The majority of the HIV-positive patients were on ART at the time of the ACS event $(n=20 / 30 ; 66.7 \%)$, while eight $(n=8 / 30$;
26.7\%) were ART-naïve and two $(n=2 / 30 ; 6.7 \%)$ had defaulted their medication at the time of the event. Of the HIV-positive patients receiving ART, $15(n=15 / 20$; $75 \%)$ were on a non-nucleoside reverse transcriptase inhibitor (NNRTI) - based regimen and five $(n=5 / 20$; $25 \%$ ) were on a protease inhibitor (PI) - based regimen.

\section{Management}

There was no difference in the immediate or long-term management of the patients (Table 3). Rates of thrombolysis in patients with STEMIs did not differ significantly between HIV-positive and HIV-negative patients.

\section{Discussion}

In this retrospective cross-sectional analysis of patient records at a large tertiary hospital in Cape Town, South Africa, the 12-month period prevalence of HIV in those hospitalised with ACS was 3.4\% (95\% CI 2.34.8). HIV-positive patients were younger, predominantly male, had a lower prevalence of medical comorbidities and a lower prevalence of a family history of CAD, even after adjusting for age and sex. HIVpositive patients experienced more STEMIs and singlevessel disease. Our findings contribute data on the prevalence and presentation of HIV in ACS from South Africa with the largest ART program in the world and a rising rate of non-communicable diseaseattributed morbidity and mortality [16].

The relative absence of traditional risk factors in the HIV-positive sub-group suggests additional pathogenic mechanisms in these patients; one being the higher rates of sub-clinical dyslipidaemia seen in HIV-positive individuals. HIV autopsy studies have shown evidence of premature CAD in HIV-positive patients even before initiation of ART, as a result of complex dyslipidaemic patterns: reduced total serum cholesterol, reduced high-density lipoprotein (HDL), reduced apolipoprotein $\mathrm{B}$, and increased low-density lipoprotein (LDL) [4]. Despite a lower prevalence of diagnosed dyslipidaemia in our HIV-positive sub-group, we do not have data on their lipid profiles at the time of presentation for ACS,

Table 2 ACS presentation of HIV-positive compared to HIV-negative patients (crude and adjusted)

\begin{tabular}{|c|c|c|c|c|c|}
\hline Outcome, n (\%) & $\mathrm{HIV}+(n=30)$ & HIV- $(n=90)$ & Crude OR (95\% Cl) & aaOR $(95 \% \mathrm{Cl})$ & Adjusted $p$-value \\
\hline \multicolumn{6}{|l|}{ ACS type } \\
\hline STEMI & $23(76.7 \%)$ & $48(53.3 \%)$ & $2.88(1.12-7.37)$ & $3.12(1.16-8.40)$ & 0.024 \\
\hline NSTEMI (reference) & $7(23.3 \%)$ & $32(35.6 \%)$ & & & \\
\hline UA (reference) & $0(0.00)$ & $10(8.3 \%)$ & & & \\
\hline \multicolumn{6}{|l|}{ Single/multi-vessel disease } \\
\hline Single-vessel & $16(53.3 \%)$ & 19 (21.1\%) & $4.27(1.78-10.27)$ & $3.03(1.16-7.96)$ & 0.024 \\
\hline Multi-vessel (reference) & $14(46.7 \%)$ & 71 (78.9\%) & & & \\
\hline
\end{tabular}

adjusted odds ratio conditioned on age and sex. Bolded text = statistically significant. OR odds ratio, CI confidence interval, ACS acute coronary syndrome, STEMI ST-elevation myocardial infarction, NSTEMI non-ST-elevation myocardial infarction, UA unstable angina 
Table 3 Angiographic data and management of HIV-positive compared to HIV-negative patients

\begin{tabular}{|c|c|c|c|}
\hline Outcome, n (\%) & $\mathrm{HIV}+(n=30)$ & HIV- $(n=90)$ & $p$-value \\
\hline \multicolumn{4}{|l|}{ Culprit coronary artery } \\
\hline Left main stem & $0(0.0 \%)$ & $2(2.2 \%)$ & 1 \\
\hline Left anterior descending & $16(53.3 \%)$ & $38(42.2 \%)$ & 0.299 \\
\hline Diagonal (1st branch) & $1(3.3 \%)$ & $5(5.6 \%)$ & 1.000 \\
\hline Circumflex & $4(13.3 \%)$ & $8(8.9 \%)$ & 0.492 \\
\hline Oblique marginal (1st branch) & $1(3.3 \%)$ & $3(3.3 \%)$ & 1.000 \\
\hline Right coronary artery & $6(20.0 \%)$ & $25(27.8 \%)$ & 0.476 \\
\hline Other & $1(3.3 \%)$ & $1(1.1 \%)$ & 0.439 \\
\hline \multicolumn{4}{|l|}{ Region affected by STEMI } \\
\hline Antero-septal & $13(56.5 \%)$ & $26(54.2 \%)$ & 1 \\
\hline Lateral & $9(39.1 \%)$ & $11(22.9 \%)$ & 0.171 \\
\hline High-lateral & $1(4.4 \%)$ & $4(8.3 \%)$ & 1 \\
\hline Inferior & $9(39.1 \%)$ & $22(45.8 \%)$ & 0.620 \\
\hline Posterior & $2(8.7 \%)$ & $8(16.7 \%)$ & 0.482 \\
\hline Right ventricle & $1(4.4 \%)$ & $2(4.2 \%)$ & 1 \\
\hline Other & $0(0.0 \%)$ & $1(2.1 \%)$ & 1 \\
\hline \multicolumn{4}{|l|}{ Radiological investigations } \\
\hline Echocardiogram & $22(73.3 \%)$ & 77 (85.6\%) & 0.165 \\
\hline MRI & $1(3.3 \%)$ & $6(6.7 \%)$ & 0.670 \\
\hline \multicolumn{4}{|l|}{ Intervention in those with STEMI } \\
\hline Thrombolysis & $17(73.9 \%)$ & $30(62.5 \%)$ & 0.341 \\
\hline $\mathrm{PCl}$ & $16(69.6 \%)$ & $38(79.2 \%)$ & 0.375 \\
\hline Conservative & $2(8.7 \%)$ & $5(10.4)$ & 0.820 \\
\hline \multicolumn{4}{|l|}{ Discharge medication } \\
\hline Antiplatelet & $30(100.0 \%)$ & $90(100.0 \%)$ & 1 \\
\hline Angiotensin converting enzyme-inhibitor & $26(86.7 \%)$ & 79 (87.8\%) & 1 \\
\hline Angiotensin receptor blocker & $1(3.3 \%)$ & $7(7.8 \%)$ & 0.678 \\
\hline Beta-blocker & $30(100.0 \%)$ & $86(95.6 \%)$ & 0.571 \\
\hline Statin & $28(93.3 \%)$ & $90(100.0 \%)$ & 0.061 \\
\hline Simvastatin & $25(92.6 \%)$ & $88(97.8 \%)$ & 0.227 \\
\hline Atorvastatin & $2(7.4 \%)$ & $2(2.2 \%)$ & 0.227 \\
\hline Nitrate & $2(6.7 \%)$ & $9(10.0 \%)$ & 0.729 \\
\hline Calcium-channel blocker & $0(0.0 \%)$ & $6(6.7 \%)$ & 0.335 \\
\hline Thiazide diuretic & $0(0.0 \%)$ & $7(7.8 \%)$ & 0.190 \\
\hline Loop diuretic & $5(16.7 \%)$ & $17(18.9 \%)$ & 1 \\
\hline Potassium-sparing diuretic & $1(3.3 \%)$ & $1(1.1 \%)$ & 0.439 \\
\hline
\end{tabular}

STEMI ST-elevation myocardial infarction, $M R I$ magnetic resonance imaging, $P C l$ percutaneous coronary intervention

which may have revealed a higher prevalence of subclinical dyslipidaemia. We did not find an association between HIV-positive patients suffering from ACS and those receiving PIs which makes it plausible that there is another risk factor apart from PIs in the causation of dyslipidaemia and ACS. Our sample size is however limited to draw definitive conclusions. The cardiovascular protective effect of ART however is supported by early ART-initiation to prevent CAD in HIV-positive individuals, as well as the increased risk of CAD and other comorbidities associated with ART-interruption [17]. Therefore, it is plausible that it is the combined effect of HIV infection and certain ART drugs that results in complex sub-clinical dyslipidaemic patterns in HIV-positive patients, thereby increasing their risk for CAD and resultant ACS. 
Table 4 Echocardiographic parameters of HIV-positive compared to HIV-negative patients

\begin{tabular}{llll}
\hline Variable, mean (SD) except where specified & HIV+ & HIV- & p-value \\
\hline LA diameter $(\mathrm{mm})(n=48)$ & $3.47(0.52)$ & $3.84(0.67)$ & 0.124 \\
LA area $\left(\mathrm{cm}^{2}\right)(n=73)$ & $17.89(4.28)$ & $19.58(4.80)$ & 0.219 \\
LVIDs $(\mathrm{mm})(n=73)$ & $\mathbf{3 . 3 7}(\mathbf{0 . 5 4})$ & $\mathbf{3 . 9 6}(\mathbf{0 . 9 0 )}$ & $\mathbf{0 . 0 1 9}$ \\
LVIDd $(\mathrm{mm})(n=77)$ & $4.72(0.49)$ & $5.11(0.83)$ & 0.083 \\
Ejection fraction (\%) $(n=86)$ & $45.4 \%(11.56)$ & $44.3 \%(12.46)$ & 0.758 \\
E/e' $(n=69)$ & $12.18(5.06)$ & $14.06(8.22)$ & 0.4171 \\
Effusion: $\mathrm{n}(\%)(n=77)$ & $2(13.3 \%)$ & $7(11.3 \%)$ & 1 \\
\hline
\end{tabular}

Bolded text = statistically significant. SD standard deviation, LA left atrium, LVIDs left ventricular internal diameter end-systole, LVIDd left ventricular internal dimension end-diastole, $E / e^{\prime}$ ratio between early mitral inflow velocity and mitral annular early diastolic velocity

An additional suggested pathogenic mechanism for CAD in HIV-positive individuals is immune-system dysfunction, which can be directly measured by the number of $\mathrm{CD}^{+}$lymphocytes in the body. Lichtenstein et al. showed that a CD4 $4^{+}$count of less than 500 cells $/ \mathrm{mm}^{3}$ is an independent risk factor for $\mathrm{CAD}$, with comparative attributable risk of approximately $20 \%$ - a figure similar to several other traditional CAD risk factors [18]. The majority $(81 \%)$ of patients in our study had $\mathrm{CD} 4^{+}$counts less than 500 cells $/ \mathrm{mm}^{3}$ which suggests an increased risk of CAD in these patients. Current detectable viraemia has been found to be a further risk factor for CAD, due to its contribution to an environment of persistent chronic inflammation [17]. Even virally suppressed HIVpositive patients have higher levels of inflammatory markers than those without HIV; thereby predisposing them to CAD [8].

The predominant presentation of single-vessel CAD and STEMIs in the HIV-positive patients in our study (even after adjusting for age and sex) is most likely due to the unique histological characteristics of coronary

Table 5 Immunological and ART status of HIV-positive patients

\begin{tabular}{ll}
\hline Variable, $\mathbf{n}(\%)$ except where specified & HIV+ \\
\hline Viral load (copies/mL) $(n=26)$ & $17(65.4 \%)$ \\
$\quad$ Suppressed $(<200)$ & $9(34.6 \%)$ \\
Unsuppressed $(>200)$ & \\
CD4 ${ }^{+}$count (cells $\left./ \mathrm{mm}^{3}\right)(n=27)$ & $271(355)$ \\
Median (IQR) & \\
ART status $(n=30)$ & $20(66.7 \%)$ \\
Treated with ART at time of event & $8(26.7 \%)$ \\
ART-naïve & $2(6.7 \%)$ \\
Defaulted ARTs & \\
ART regimen ( $n=20)$ & $14(70.0 \%)$ \\
Tenofovir-emtricitibine-efavirenz & $1(5.0 \%)$ \\
Abacavir-lamivudine-efavirenz & $4(20.0 \%)$ \\
Zidovudine-lamivudine-lopinavir/ritonavir & $1(5.0 \%)$ \\
\hline Stavudine-lamivudine-lopinavir/ritonavir &
\end{tabular}

IQR inter-quartile range, $A R T$ anti-retroviral therapy plaques in these patients. Virtual histology intravascular ultrasound analysis of HIV-positive patients affected by ACS has shown a high prevalence of unstable plaque morphology that is rich in necrotic tissue, less calcific, and has a thicker fibrous cap compared to that seen in traditional CAD [4]. Non-calcified plaques are more likely to rupture than calcific or mixed plaques, putting these patients at higher risk of single-vessel STEMI than their HIV-negative counterparts [19]. This higher plaque vulnerability is thought to be linked to the chronic inflammatory process of being infected with the HI virus itself. Furthermore, Moran et al. found that HIV-positive patients with single-vessel disease had higher Gensini Scores [20] than HIV-negative controls with singlevessel disease, indicating more severe vessel stenosis in the former [19]. The presence of more extensive, more vulnerable non-calcific, fibro-fatty plaque could also explain the higher prevalence and earlier onset of ACS in the HIV-positive population.

The mild systolic dysfunction seen in both groups is in keeping with the echocardiographic changes expected after a myocardial infarction. We would expect more advanced diastolic dysfunction in our HIV-negative subgroup as a result of the increased prevalence of hypertension in this group. A study done in Cameroon comparing the left atrial remodelling in hypertensives compared to healthy patients showed the hypertensive patients to have a larger left atrial diameter, surface area and volume, indicating an altered diastolic function in these patients [21]. Although statistically nonsignificant, it is plausible that the $11 \mu \mathrm{mol} / \mathrm{L}$ higher mean serum creatinine in the HIV-negative sub-group can be explained by the increased prevalence of medical comorbidities in this group, resulting in a higher incidence of target-organ damage which may manifest as subclinical/clinical chronic kidney disease. Serum creatinine is reported in many studies as a prognostic marker for overall cardiac mortality. In a study by Matts et al. it was found that each nine $\mu \mathrm{mol} / \mathrm{L}(0.1 \mathrm{mg} / \mathrm{dL})$ rise in baseline serum creatinine had a $36 \%$ increased relative risk of future overall mortality and a $47 \%$ increased relative risk 
for future atherosclerotic CAD mortality (no confounding factors present) [22]. This potentially translates into a $44 \%$ increased relative risk of overall future mortality and a 57\% increased relative risk for future atherosclerotic CAD mortality in the HIV-negative patients in our study. Such a finding in our study cannot be overinterpreted due to our small sample size.

As there is no current evidence for a change in immediate or long-term management of CAD and ACS based on HIV status, all patients in our study were treated similarly. Simvastatin coadministered with PIs are expected in increase simvastatin concentrations markedly due to CYP3A inhibition [23]. However, we noted that $80 \%$ of patients on PIs incorrectly received simvastatin. The more frequent prescription of calcium-channel blockers and thiazide diuretics in the HIV-negative subgroup is most likely as a result of the increased prevalence of hypertension in this population.

Overall, our findings were consistent with the literature. There is limited global data on the prevalence of HIV in people presenting with ACS, but our findings are similar to the $2.4 \%$ HIV prevalence found in the CAD sub-group (consisting of 581 patients) of all de novo cases of heart disease presenting to a tertiary hospital in Soweto, South Africa [24]. These values are significantly lower than both the $13.1 \%$ estimated national HIVprevalence in South Africa in 2018 and the 7\% prevalence of HIV in a population of people with ACS in a HIC such as Spain [11]. This may be due to the underreporting of HIV in ACS in South Africa as evidenced by the large portion of untested patients in our study (Fig. 1) [1]. Furthermore, it may be due to other confounding factors; one being age: HIV-prevalence is highest in young adults whereas ACS primarily affects the elderly. Despite this, data from a study conducted in Québec, Eastern Canada, showed the incidence of ACS to be 3.88 in the HIV-positive cohort compared to 2.21 in the HIV-negative cohort per 1000 patient-years, irrespective of exposure to ART [25]. A study conducted in Boston, Massachusetts, showed increased ACS rates per 1000 person-years in HIV-positive vs HIV-negative patients (11.13 vs 6.98), even after adjusting for age, sex, race, hypertension, diabetes and dyslipidaemia [12]. The demographic profile and CAD risk factor profile of the patients in our study was consistent with that seen in the literature. Furthermore, the type of ACS and number of involved coronary arteries of the HIV-positive patients in our study matched that seen in the literature. Various studies from the USA and France showed angiographic findings of fewer involved vessels and a greater burden of inflammatory plaque in the HIV-positive cases as compared to the HIV-uninfected cases [7]. Our findings of a predilection for LAD artery involvement in the HIV-positive patients are supported by those of Vachiat et al. who showed the LAD to be the most common culprit artery (60\%) in HIV-positive patients with ACS [26]. The same proportion of HIV-positive and HIVnegative patients in our study underwent percutaneous coronary intervention (PCI), but we have no follow-up data on long-term success rates of such an intervention. A Spanish study showed a lower longterm success-rate in HIV-positive patients who underwent PCI compared to HIV-negative patients (75\% versus $85 \%$ success-rate respectively) [27].

An interesting finding from our study was that despite a nonsignificant increased unadjusted prevalence of smoking seen in HIV-positive compared to HIVnegative patients, this finding was reversed when adjusting for age and sex. This finding could perhaps explain the heterogeneity in smoking data when comparing HIV-positive and HIV-negative patients with ACS. The majority of studies show a positive correlation between HIV-infection and smoking; however, when matching on age and sex, Dwyer et al found no difference in rates of smoking between HIV-positive and HIV-negative subgroups [1,28].

Our study has a number of limitations. First, the retrospective, observational design limits its ability to control for unmeasured confounders. Second, our sample size is limited which did not allow us to perform sub-group analyses. Third, we may have underestimated the HIVprevalence within the ACS population due to the relative infrequent reporting of HIV-results. It is not routine for everyone presenting to a healthcare facility in South Africa to be tested for HIV, meaning that the actual prevalence of HIV within the ACS population might be higher than reported in our study. Fourth, our sample is from a single centre and may not be representative of South Africa or sub-Saharan Africa. Last, we were confronted with missing data that were not always collected as part of routine clinical care. $\mathrm{CD} 4^{+}$counts and viral loads were not available for all HIV-positive patients, and when available, were not always taken during the same admission as for the ACS event. It is also possible that we may have missed cases with our retrospective design. However, given that we used electronic database systems for our case identification and data extraction, we argue that this risk was minimised.

\section{Conclusions}

In conclusion, we found that HIV-positive patients with ACS were younger, more likely to present with STEMIs and single-vessel disease, and had limited traditional ACS risk factors. This suggests additional pathogenic mechanisms for the development of CAD and ACS in patients with HIV; possibly attributed to the inflammation and immune-activation caused by infection with HIV. To date, ART is the most important treatment 
intervention highlighting the importance of prompt initiation of ART in patients with HIV [29]. The younger age of ACS presentation in patients with HIV highlights the importance of early, rigorous screening for risk factors and early diagnosis of CAD for active management. Our prevalence finding of $3.4 \%$ of HIV in ACS may be an under-estimate in South Africa and should be prospectively studied.

\begin{abstract}
Abbreviations
CAD: Coronary artery disease; HIC: High-income country; ACS: Acute coronary syndrome; TBH: Tygerberg Hospital; SSA: Sub-Saharan Africa; Cl: Confidence interval; STEMI: ST-elevation myocardial infarction; OR: Odd's ratio; ART: Anti-retroviral therapy; LMIC: Low/middle-income country; NSTE MI: Non-ST-elevation myocardial infarction; UA: Unstable angina; ECG: Electrocardiogram; ECM: Electronic Content Management System; MI: Myocardial infarction; NHLS: National Health Laboratory Service; SD: Standard deviation; LAD: Left anterior descending; LVIDs: Left ventricular internal dimension at end-systole; VL: Viral load; NNRTI: Non-nucleoside reverse transcriptase inhibitor; PI: Protease inhibitor; HDL: High-density lipoprotein; LDL: Low-density lipoprotein; PCl: Percutaneous coronary intervention
\end{abstract}

\section{Acknowledgements}

The authors wish to thank Veshni Pillay-Fuentes Lorente for her help creating the study database and for her support and guidance throughout the project. We also acknowledge Pieter-Paul Robertse for his contribution to the study design.

\section{Authors' contributions}

ED conceived the study and edited the manuscript. CP developed the study protocol, managed data collection and coding, and was a major contributor in writing the manuscript. TE analysed the patient data. AD interpreted the data and contributed to editing the manuscript. All authors read and approved the final manuscript.

\section{Funding}

No external funding was utilised.

\section{Availability of data and materials}

The datasets used and/or analysed during the current study are available from the corresponding author on reasonable request.

\section{Declarations}

\section{Ethics approval and consent to participate}

Ethical approval for the study as well as a waiver of consent for access of clinical/personal data was obtained from the Human Research Ethics Committee of the Faculty of Medicine and Health Sciences of Stellenbosch University (Reference number: U19/05/023). This waiver negated the need for acquisition of any administrative permissions or licenses.

\section{Consent for publication}

Not applicable.

\section{Competing interests}

The authors declare that they have no competing interests.

\section{Author details}

'Division of Clinical Pharmacology, Department of Medicine, Faculty of Medicine and Health Sciences, Stellenbosch University and Tygerberg Hospital, PO Box 241, Cape Town 8000, Republic of South Africa. ${ }^{2}$ Division of Epidemiology and Biostatistics, Department of Global Health, Faculty of Medicine and Health Sciences, Stellenbosch University, PO Box 241, Cape Town 8000, Republic of South Africa. ${ }^{3}$ Division of Cardiology, Department of Medicine, Faculty of Medicine and Health Sciences, Stellenbosch University and Tygerberg Hospital, PO Box 241, Cape Town 8000, Republic of South Africa.
Received: 29 June 2020 Accepted: 28 June 2021

Published online: 07 July 2021

\section{References}

1. Calvo M, Saubí N, Gatell JM, Miró O, Sánchez M, Foix A, et al. Clinical presentation of acute coronary syndrome in HIV infected adults: a retrospective analysis of a prospectively collected cohort. Eur J Intern Med. 2011;22(5):485-8. https://doi.org/10.1016/j.ejim.2011.02.017.

2. Boccara F, Lang S, Meuleman C, Ederhy S, Mary-Krause M, Costagliola D, et al. HIV and coronary heart disease: time for a better understanding. J Am Coll Cardiol. 2013;61(5):511-23. https://doi.org/10.1016/j.jacc.2012.06.063.

3. Boccara F. Acute coronary syndrome in HIV-infected patients. Does it differ from that in the general population? Arch Cardiovasc Dis. 2010;103(11-12): 567-9. https://doi.org/10.1016/j.acvd.2010.10.004.

4. Seecheran VK, Giddings SL, Seecheran NA. Acute coronary syndromes in patients with HIV. Coron Artery Dis. 2017;28(2):166-72. https://doi.org/10.1 097/MCA.0000000000000450.

5. Khunnawat C, Mukerji S, Havlichek D, Touma R, Abela GS. Cardiovascular manifestations in human immunodeficiency virus-infected patients. Am J Cardiol. 2008;102(5):635-42. https://doi.org/10.1016/j.amjcard.2008.04.035.

6. Strijdom H, De Boever P, Walzl G, Essop MF, Nawrot TS, Webster I, et al. Cardiovascular risk and endothelial function in people living with HIV/AIDS: design of the multi-site, longitudinal EndoAfrica study in the Western Cape Province of South Africa. BMC Infect Dis. 2017;17(1):1-9. https://doi.org/1 0.1186/s12879-016-2158-y.

7. Hyle EP, Mayosi BM, Middelkoop K, Mosepele M, Martey EB, Walensky RP, et al. The association between HIV and atherosclerotic cardiovascular disease in sub-Saharan Africa: a systematic review. BMC Public Health. 2017; 17(1):1-15. https://doi.org/10.1186/s12889-017-4940-1.

8. Bahrami H, Budoff M, Haberlen SA, Rezaeian P, Ketlogetswe K, Tracy R, et al. Inflammatory markers associated with subclinical coronary artery disease: the multicenter AIDS cohort study. J Am Heart Assoc. 2016;5(6):1-13. https://doi.org/10.1161/JAHA.116.003371.

9. Vedanthan R, Seligman B, Fuster V, Weiner MA, Kravis HR. Global perspective on acute coronary Sydrome: a burden on the young and poor. Circ Res. 2014;114(12):1959-75. https://doi.org/10.1161/CIRCRESAHA.114.302782.

10. Maluleke R. Mortality and causes of death in South Africa, 2016: Findings from death notification. 2016. Available from: https:/www.statssa.gov.za/ publications/P03093/P030932016.pdf. Accessed 22 May 2020.

11. Stats SA. Mid-year population estimates 2018. Pretoria; 2018. Available from: http://www.statssa.gov.za/publications/P0302/P03022018.pdf. Accessed 23 June 2019.

12. Triant VA, Lee H, Hadigan C, Grinspoon SK. Increased acute myocardial infarction rates and cardiovascular risk factors among patients with human immunodeficiency virus disease. J Clin Endocrinol Metab. 2007;92(7):250612. https://doi.org/10.1210/jc.2006-2190.

13. WellSky International Ltd. JAC Computer Services Ltd. 2009. Available from: https://www.wellsky.org/. Accessed 23 Sept 2019.

14. Thygesen K, Alpert JS, Jaffe AS, Chaitman BR, Bax JJ, Morrow DA, et al. Fourth universal definition of myocardial infarction. J Am Coll Cardiol. 2018; 72(18):2231-64. https://doi.org/10.1016/j.jacc.2018.08.1038.

15. National Department of Health SA. Standard treatment guidelines and essential medicines list for south africa: hospital level. 5th ed; 2019. https:// doi.org/10.1017/CBO9781107415324.004.

16. Elflein J. Ranking of countries with the highest prevalence of HIV in 2000 and 2018. 2019. Available from: https:/www.statista.com/statistics/270209/ countries-with-the-highest-global-hiv-prevalence/. Accessed 22 Apr 2020.

17. Lacson J, Barnes R, Bahrami H. Coronary artery disease in HIV-infected patients: downside of living longer. Curr Atheroscler Rep. 2017;19(4):18. https://doi.org/10.1007/s11883-017-0651-4.

18. Lichtenstein KA, Armon C, Buchacz K, Chmiel JS, Buckner K, Tedaldi EM, et al. Low CD4 + T cell count is a risk factor for cardiovascular disease events in the HIV outpatient study. Clin Infect Dis. 2010;51(4):435-47. https://doi.org/10.1086/655144.

19. Moran CA, Southmayd G, Devireddy CM, Quyyumi AA, Ofotokun I, Liberman HA, et al. Clinical and procedural characteristics of persons living with HIV presenting with acute coronary syndrome. AIDS. 2020;34(1):83-90. https:// doi.org/10.1097/QAD.0000000000002393.

20. Rampidis GP, Benetos G, Benz DC, Giannopoulos AA, Buechel RR. A guide for Gensini Score calculation. Atherosclerosis. 2019;287:181-3. https://doi. org/10.1016/j.atherosclerosis.2019.05.012. 
21. Gounoue AC, Tamdja AD, Choukem SP, Ba H, Aboubakar SM, et al. Echocardiographic left atrial remodelling and determinants of left atrial size in the early phase of high blood pressure: a comparative cross-sectional study in Douala, Cameroon. Cardiovasc J Afr. 2020;31(1):40-6. https://doi. org/10.5830/CVJA-2019-046.

22. Matts JP, Karnegis JN, Campos CT, Fitch LL, Johnson JW, Buchwald H. Serum creatinine as an independent predictor of coronary heart disease mortality in normotensive survivors of myocardial infarction. POSCH Group. J Fam Pract. 1993;36(5):497-503.

23. Chastain DB, Stover KR, Riche DM. Evidence-based review of statin use in patients with HIV on antiretroviral therapy. J Clin Transl Endocrinol. 2017;8: 6-14. https://doi.org/10.1016/j.jcte.2017.01.004

24. Sliwa K, Carrington MJ, Becker A, Thienemann F, Ntsekhe M, Stewart S. Contribution of the human immunodeficiency virus/acquired immunodeficiency syndrome epidemic to de novo presentations of heart disease in the heart of Soweto study cohort. Eur Heart J. 2012;33(7):866-74. https://doi.org/10.1093/eurheartj/ehr398.

25. Durand M, Sheehy O, Baril J, Lelorier J, Tremblay L. Association between HIV infection, Antiretroviral Therapy, and risk of Acute Myocardial Infarction: A cohort and nested case - control study using Quebec's health insurance database. J Acquir Immune Defic Syndr. 2011;57(3):245-53. https://doi.org/1 0.1097/QAl.0b013e31821d33a5.

26. Vachiat A, McCutcheon K, Tsabedze N, Zachariah D, Manga P. Atherosclerotic plaque in HIV-positive patients presenting with acute coronary syndromes. Cardiovasc J Afr. 2019;30(4):203-7. https://doi.org/10. 5830/CVJA-2019-016

27. Martin-Reyes R, Galeote G, Moreno R, Sanchez-Recalde A, Lopez De SE, Lopez-Sendon J. Percutaneous coronary interventionism in patients infected with human immunodeficiency virus admitted for acute coronary syndrome: a case- control study. Med Clin. 2010;24:4-6. https://doi.org/10.1 016/j.medcli.2010.05.008.

28. O'Dwyer EJ, Bhamra-Ariza P, Rao S, Emmanuel S, Carr A, Holloway CJ. Lower coronary plaque burden in patients with HIV presenting with acute coronary syndrome. Open Heart. 2016;3(2):511-7. https://doi.org/10.1136/ openhrt-2016-000511.

29. Boccara F, Cohen A. HIV and heart disease: What cardiologists should know. Rev Española Cardiol. 2016;69:1126-30. https://doi.org/10.1016/j.rec.2016.05. 032.

\section{Publisher's Note}

Springer Nature remains neutral with regard to jurisdictional claims in published maps and institutional affiliations.

\section{Ready to submit your research? Choose BMC and benefit from:}

- fast, convenient online submission

- thorough peer review by experienced researchers in your field

- rapid publication on acceptance

- support for research data, including large and complex data types

- gold Open Access which fosters wider collaboration and increased citations

- maximum visibility for your research: over $100 \mathrm{M}$ website views per year

At $\mathrm{BMC}$, research is always in progress.

Learn more biomedcentral.com/submissions 